\title{
Study of the anisotropy of cosmic rays during the periods of the minima of the 24th solar cycle using the muonography method according to the data of the URAGAN muon hodoscope
}

\section{I.I. Yashin ${ }^{a, b}$, I.I. Astapov ${ }^{a}$, A.N. Dmitrieva ${ }^{a, b}$, A.G. Gvishiani ${ }^{b}$, V.G. Getmanov ${ }^{b}$,} A.A. Kovylyaeva $^{a, b}$, Yu. N. Mishutina ${ }^{a, b}$, A.A. Soloviev ${ }^{b}$ and V.V. Shutenko ${ }^{a}$

${ }^{a}$ National Research Nuclear University MEPhI (Moscow Engineering Physics Institute), Kashirskoe shosse 31, Moscow, Russia

${ }^{b}$ Geophysical Center of Russian Academy of Sciences (GC RAS),

Molodezhnaya St. 3, Moscow, Russia

E-mail: iiyashin@mephi.ru

\begin{abstract}
Muon hodoscope URAGAN (MEPhI, Moscow) with an area of 45 sq. $\mathrm{m}$ is capable of real time detection of the tracks of all muons arriving from the upper celestial hemisphere with a high spatial and angular accuracy $(1 \mathrm{~cm}$ and 1 degree, respectively). The measured angular distribution of the muons flux over a certain period of exposure time and expressed in R.M.S. deviations from an averaged reference matrix and corrected for barometric and temperature effects represents a matrix-muonograph (by analogy with X-ray radiography) of the Earth's atmosphere and near-terrestrial space. Such muonograph contains information on the current variation the flux of cosmic muons associated with modulation processes in the heliosphere, magnetosphere and atmosphere of the Earth. The sequence of muonographs converted to the GSE coordinate system allows one to study in real time the dynamics of cosmic ray anisotropy and to identify in advance geoeffective processes in the heliosphere associated with solar activity. Results of the analysis of the anisotropy of the cosmic ray muon flux at the minima of the 23rd (2009-2010) and 24th (2018-2019) solar cycles are discussed.
\end{abstract}

37th International Cosmic Ray Conference (ICRC 2021)

July 12th-23rd, 2021

Online-Berlin, Germany

"Speaker 


\section{Introduction}

During periods of low solar activity associated with solar cycle minima, recurrent phenomena of an increase in the solar wind speed are one of the main causes of disturbances in the interplanetary magnetic field, which lead to modulations of the primary fluxes of cosmic rays (CRs) passing through the Earth's magnetosphere. The main source of high-speed solar wind, which interacting with the magnetosphere generates magnetic storms, are coronal holes on the Sun [1].

Cosmic rays, as a result of interaction with atomic nuclei of the atmosphere, generate the muon component of secondary CRs, which reaches the observation level and can be detected by ground-based detectors. Since muons practically keep the direction of parent particle motion, the anisotropy of cosmic rays associated with various modulation processes in the heliosphere can be studied from the anisotropy of the muon flux. For these purposes, it is convenient to use a large-area muon hodoscope capable of registering all muons arriving from the upper hemisphere in real time. Such a detector is the URAGAN wide-aperture hodoscope $\left(55.7^{\circ} \mathrm{N}\right.$, $37.7^{\circ} \mathrm{E}, 173 \mathrm{~m}$ a.s.1.) with an area of $\sim 45 \mathrm{~m}^{2}$, which has a high spatial and angular accuracy (1 $\mathrm{cm}$ and $1^{\circ}$, respectively) [2].

URAGAN consists of four independent supermodules. Each supermodule (SM) (see Fig. 1) represents an assembly of eight planes of streamer tube chambers operated with a gas mixture $\mathrm{Ar}+\mathrm{CO}_{2}+$ n-pentane.

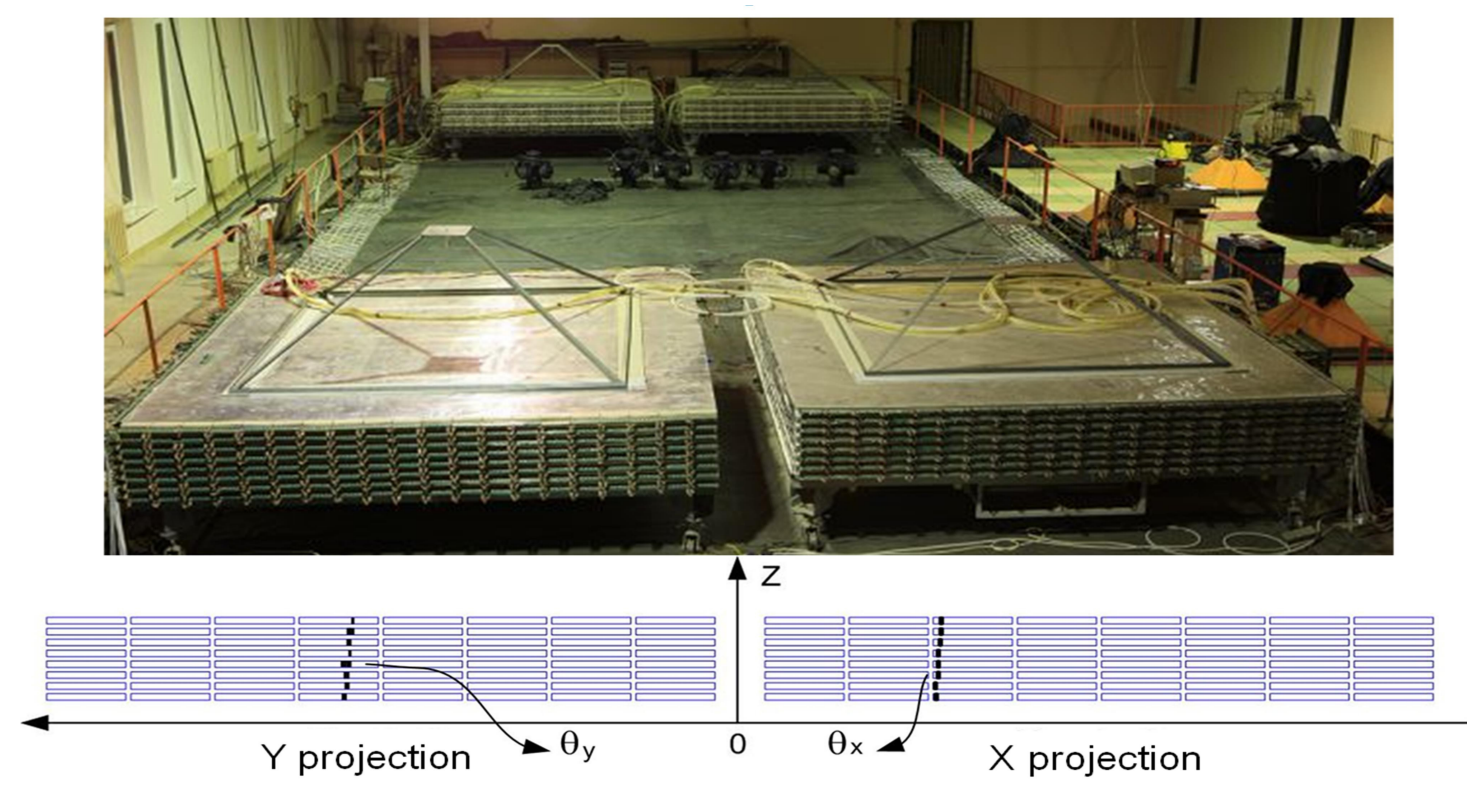

Figure 1. The muon hodoscope URAGAN. In the picture above: four supermodules are shown, below: scheme of track registration.

Sixteen gas discharge tubes, with size $9 \times 9 \times 3500 \mathrm{~mm}^{3}$ and resistive cathode coating operated in a limited streamer mode, are enclosed in a single plastic container. Each plane contains 320 tubes equipped with an external two-coordinate strip readout system. Sensitive area of one $\mathrm{SM}$ is $\sim 11 \mathrm{~m}^{2}$. Exposition is divided into equal one-minute intervals. In its turn, each interval includes actual detection (54 s), monitoring of detection channels (2 s) and transmission 
of one-minute data frame (4 s). Synchronous operation of SMs is provided by GPS/GLONASS antenna.

\section{URAGAN data format}

The angular distributions of the tracks detected during 1 min intervals are stored in three types of binary arrays-matrices with dimensions of $90 \times 90$ cells: the zenith and azimuth angles $(\theta, \varphi): M_{a} \equiv\left[\theta_{i}, \varphi_{j}\right]$, by projection angles $\left(\theta_{\mathrm{X}}, \theta_{\mathrm{Y}}\right): M_{p a} \equiv\left[\theta_{X i}, \theta_{Y j}\right]$, tangents of projection angles $\left(\operatorname{tg} \theta_{\mathrm{X}}, \operatorname{tg} \theta_{\mathrm{Y}}\right): M_{\operatorname{tg}} \equiv\left[\operatorname{tg} \theta_{X i}, \operatorname{tg} \theta_{Y j}\right]$ [2]. Every matrix stores the angular distribution of muons measured during 1-min interval. The sequence of such matrices gives unique possibility to study the temporal changing of muon angular distributions. Depending on the analysis to be performed, matrices can be collected in different time intervals. For the analysis of muon flux variations of the heliospheric origin, the matrix data summed of measuring minute intervals during 1 hour is used. In Fig. 2 shows the stages of the formation of the muonograph. In brief, a muonograph is an hourly matrix of track parameters (Fig 2a) normalized to the number of events (Fig 2(b, c)), smoothed with respect to slow trends and daily fluctuations in the intensity of the muon flux (Fig 2d), and also corrected for the shape of the angular distribution of the muon flux (Fig 2e) [2].

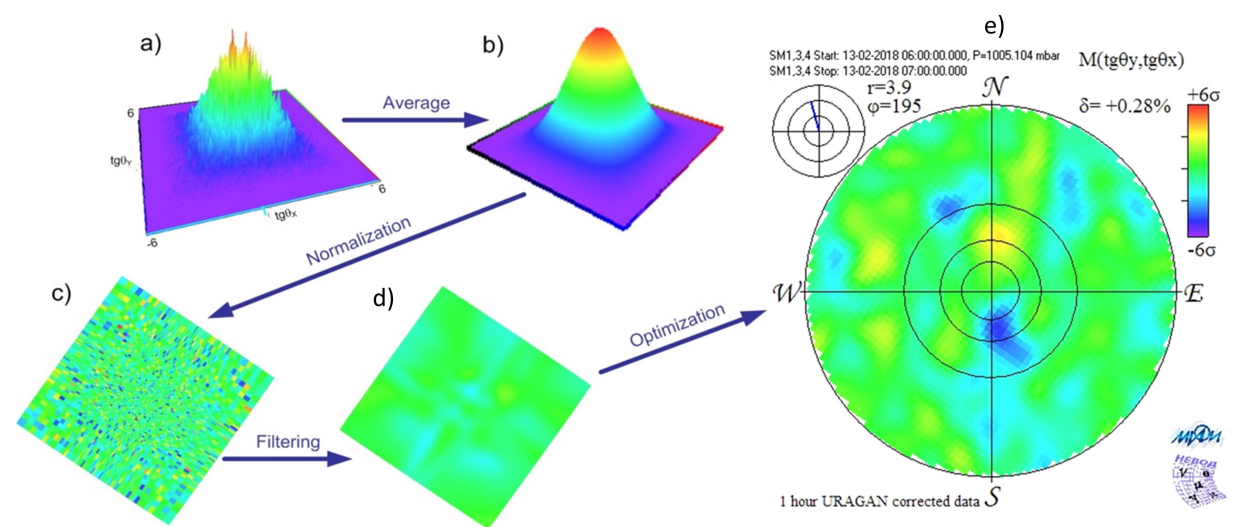

Figure 2. Stages of muonograph formation.

A graphical representation of hourly muonograph is shown in Fig. 2e. To obtain muonographs, the relative deviations of the intensities of the registered muon flux are calculated in units of statistical errors. The matrix of changes of counting rates $M_{t g_{-} \delta}$ in terms of statistical errors is calculated as follows:

$$
M_{\text {tg } \_\delta}=\frac{M_{t \mathrm{~g} \_\Delta \mathrm{t}}-M_{\mathrm{tg}}^{\mathrm{N}} \cdot t_{\text {live } \_\Delta \mathrm{t}} / t_{\text {live }}^{\mathrm{N}}}{\sqrt{M_{\mathrm{tg}}^{\mathrm{N}} \cdot t_{\text {live_ } \Delta \mathrm{t}} / t_{\text {live }}^{\mathrm{N}}}},
$$

here $t_{\text {live_t }}$ is the sum over all SMs of "live"-times of one-minute frames for time frame $\Delta \mathrm{t}=60 \mathrm{~min}, l$ is an index of the SM: 


$$
t_{\text {live_ } \Delta \mathrm{t}}=\sum_{\Delta t} \sum_{l} t_{\text {live }}^{l}
$$

Correction for barometric and temperature effects is performed before summation [3].

The sequence of such matrices makes it possible to study the temporal variation of the angular distributions of the muon flux [2]. Using asymptotic directions, muonographs from the laboratory coordinate system (LCS) are converted to the angular distribution of parent protons at the magnetopause boundary in the Geocentric Solar Ecliptic system (GSE) [4]. Figure 3 shows a diagram of the transformation of muonograph from the LCS to the GSE system.

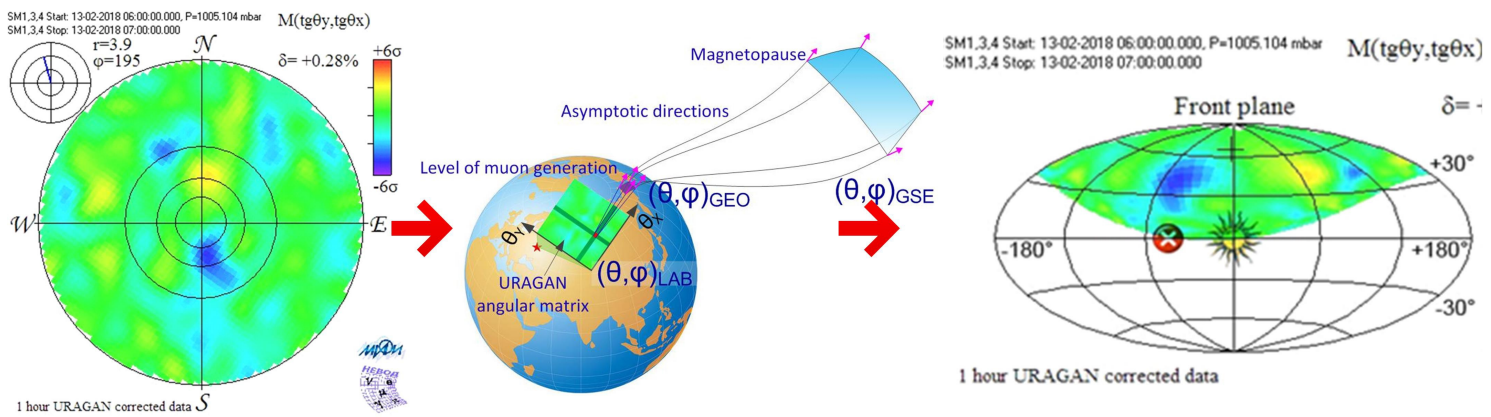

Figure 3. Muonographs of one-hour matrix in the laboratory system (left) and in the GSE coordinate system (right). The outer circle limits cells with zenith angles $75^{\circ}$. Inner circles correspond to zenith angles $30^{\circ}, 45^{\circ}$ and $60^{\circ}$.

Both in the laboratory system and in the GSE system, muonographs show the areas of deviation in the angular distribution of the flux of secondary and primary cosmic rays, namely, the size of the area (in steradians) and the deviation of the CR flux from the average over the previous day (in units of the standard deviation) [5]. Time sequences of such deviations are formed in GSE maps, which are convenient for analyzing the response of the muon hodoscope to heliospheric disturbances.

\section{GSE mapping}

On the GSE muonographs, regions with deviation $|\delta|>3 \sigma$ were selected. These regions correspond to areas of deformation (anisotropy) of the angular distribution of muons. For each deformation area the following values are calculated: solid angle and pitch angle of peak deviation in the GSE system, GSE-latitude and GSE-longitude of the peak deviations and its value in units of statistical error, peak value in percentage and its angular coordinates $(\theta, \varphi)$ in the LCS (see Fig. 4).

The obtained characteristics for each hourly muonograph in the GSE system can be plotted as points in coordinates of GSE longitudes of the peak values $(\theta, \varphi)$ versus time. Size of the points on the graphs depends on the size of the solid angle of the area, and the color on the peak value of the deviation inside the area. On the graphs, only areas with a solid angle larger than $0.02 \mathrm{sr}$ are shown. On the graphs with GSE longitude, only areas with GSE latitude less than $80^{\circ}$ are displayed. 


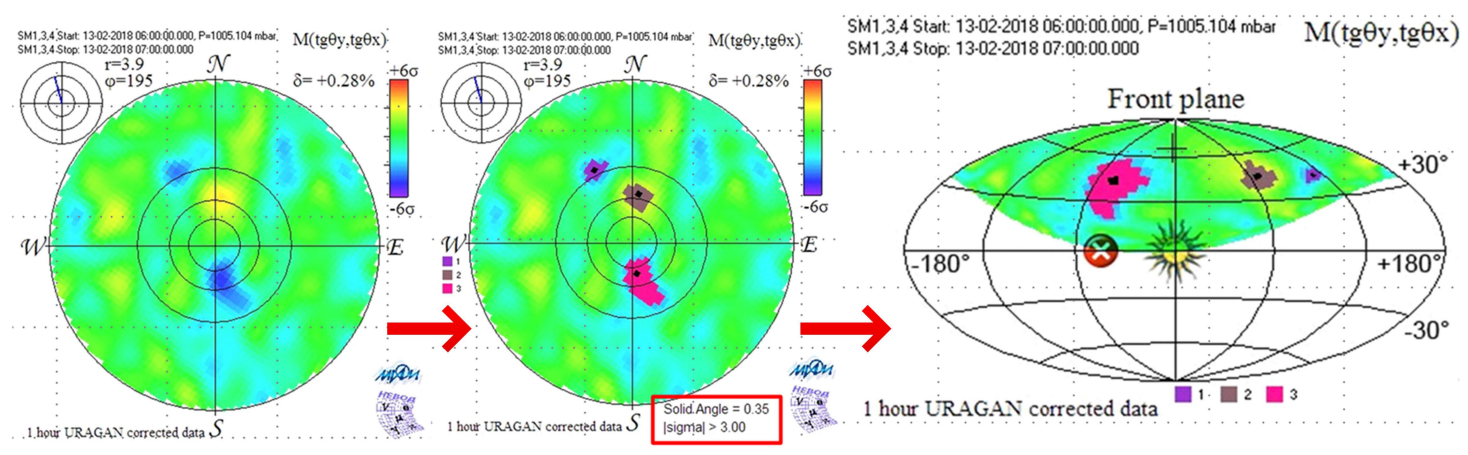

Figure 4. GSE-mappings of the muonograph. Left: the original matrix of deformations; center: the found areas of anisotropy in the muonograph; right: found areas in the GSE system.

\section{Variations of cosmic rays during minima of solar activity}

For the analysis, magnetic storms [6] that occurred during periods of minimum solar activity were selected. A total of 81 events were considered that occurred over four years: at the end of the 23rd solar cycle and the beginning of the 24th one: 2009 (15 storms), 2010 (25 storms), and 2018 (30 storms), 2019 (14 storms).

Figures 5 and 6 show the distributions of the sizes of areas and pitch angles for the 4 considered years with low solar activity. Figures represent the distributions of deformation areas (above) and pitch angles (below) for changes, both increases (pink) and decreases (violet), in the CR flux by $5 \sigma$ and more. The size of the dots corresponds to the size of the area: small dots are from 0.02 to $0.1 \mathrm{sr}$, midpoints are from 0.1 to $0.5 \mathrm{sr}$, and large dots are from 0.5 to $1 \mathrm{sr}$. The concentration and sizes of points during geomagnetic disturbances should be noted. Moreover, there is both anisotropy associated with a decrease and an increase of local intensity in angular distribution of the muon flux. Simultaneous observation of areas of increasing and decreasing flux is a distinctive feature of the hodoscopic observation method. The study of these temporal distributions and the relationship between the time of anisotropy appearance and the moment of formation of a geoeffective disturbance in the heliosphere is a separate important task.
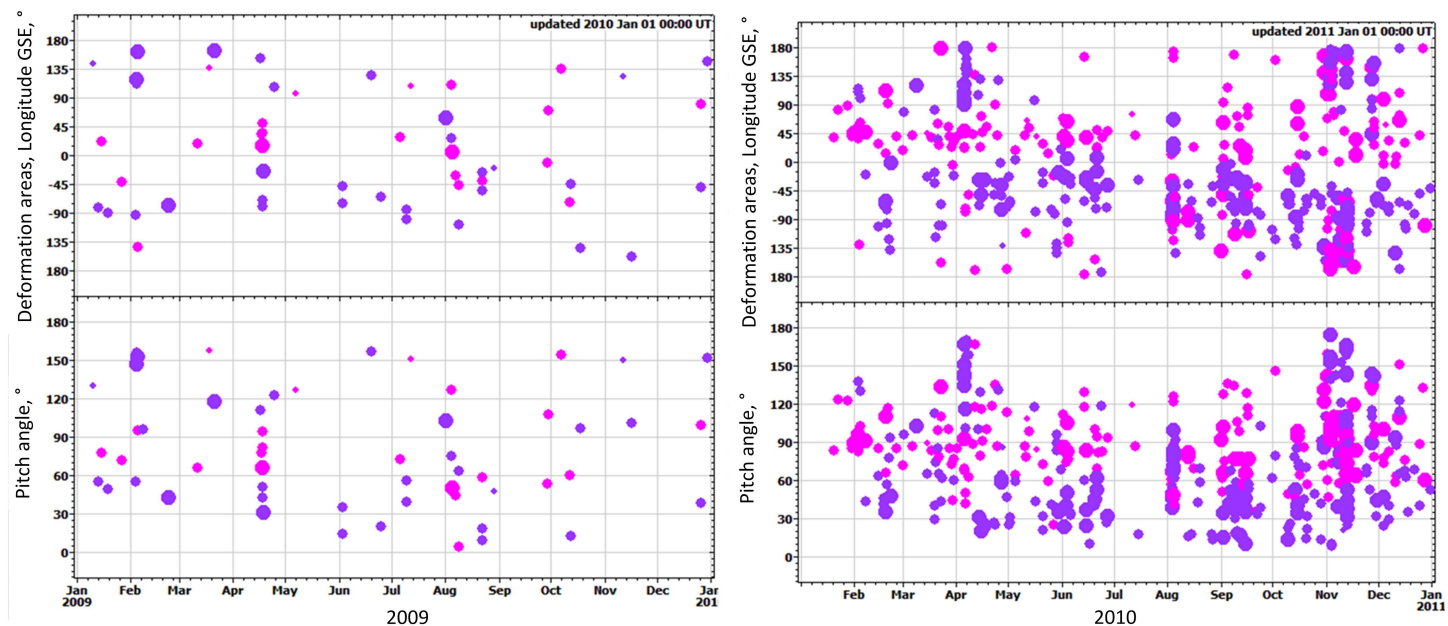

Figure 5. GSE maps for 2009 (left) and 2010 (right). 

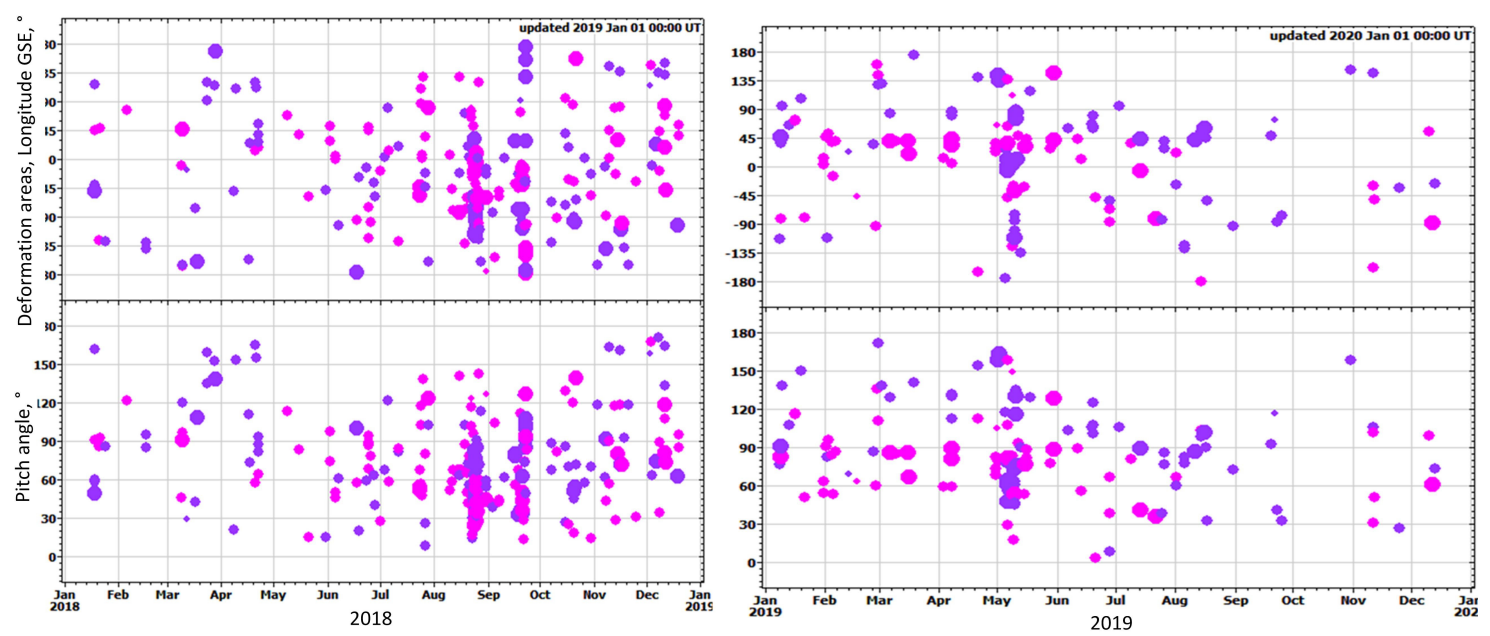

Figure 6. GSE maps for 2018 (left) and 2019 (right).

The left panel of Fig. 7 shows the distribution of the angular sizes of anisotropy by years. During this period, deformation regions with sizes ranging from 0.1 to $0.8 \mathrm{sr}$ were observed. Most often, areas of $0.4 \mathrm{sr}$ were recorded regardless of the year. The second panel in Figure 7 shows the pitch-angle distributions in the years under consideration. It was found that in 2009 the particles registered by the hodoscope most often arrived into the Earth's magnetosphere with a pitch angle of $50^{\circ}$, in 2010 with an angle of $90^{\circ}$, in 2018 of $50^{\circ}$, and in 2019 of $80^{\circ}$.
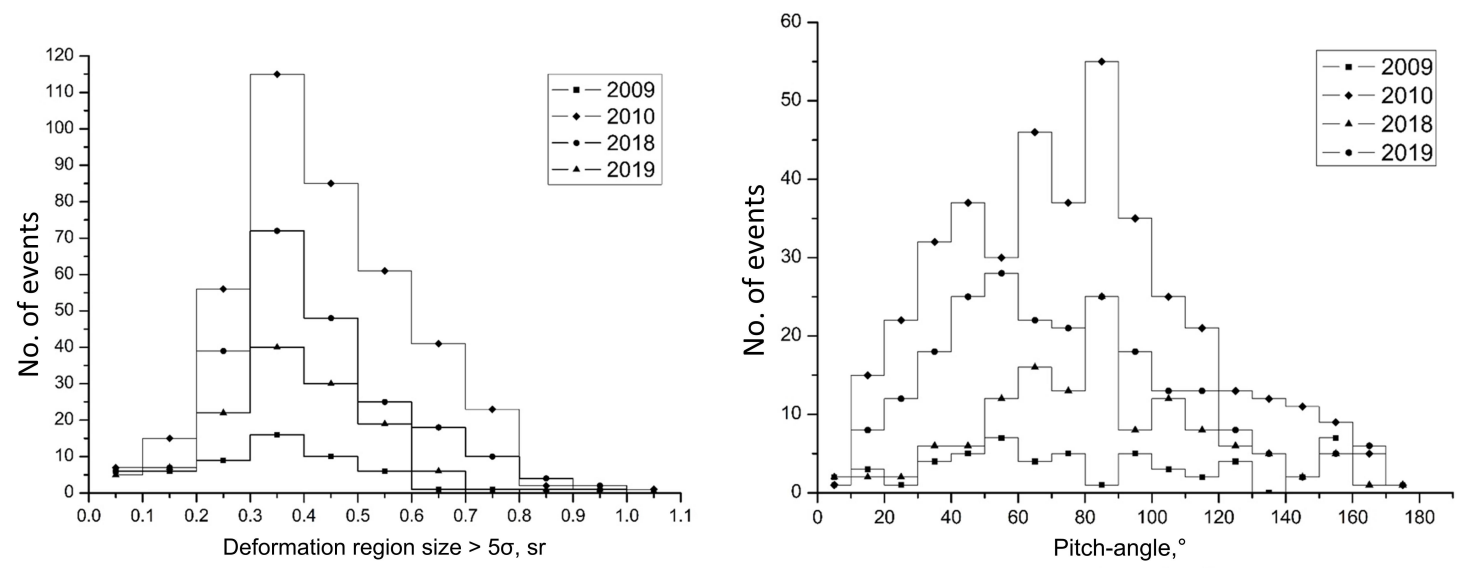

Figure 7. Distributions of the size of the regions of cosmic ray flux anisotropy (left) and the distribution of pitch angles of CR flux disturbanses detected in the years of decreased solar activity.

\section{Conclusion}

The muon hodoscope URAGAN allows us to provide a study of variations not only of integral characteristics of muon flux and its anisotropy, but also to analyze in time the changes in the angular distribution of the muon flux caused by the effects of inhomogeneities of the interplanetary magnetic field on the flux of charged primary cosmic rays.

The proposed approach of mapping the anisotropy areas in the angular distribution in a matrix form allows us to estimate the degree of deformation, the zenith and azimuthal angle of the anisotropy area center in the local coordinate system, and its GSE longitude and latitude. 
The use of the muonography method made it possible to estimate some of the features of the dynamics of the anisotropy of cosmic rays during periods of minimum solar activity with different polarities of the interplanetary magnetic field.

\section{Acknowledgments}

The work has been performed at the Unique Scientific Facility "Experimental complex NEVOD" in MEPhI with the financial support provided by the Russian Science Foundation (project No. 17-17-01215).

\section{References}

[1] I.G. Richardson. Energetic particles and corotating interaction regions in the solar wind. Space Sci. Rev., 111 (2004) 267-376.

[2] I.I. Yashin et al. Real-time data of muon hodoscope URAGAN. Adv. Space Res., 56 (2015) 26932705.

[3] A.N. Dmitrieva et al. Corrections for temperature effect for ground-based muon hodoscopes, Astropart. Phys. 34 (2011) 401-411.

[4] I.I. Yashin et al. Real-time GSE mapping of muon-flux directional variation with the URAGAN hodoscope. Bull. Rus. Acad. Sci. Phys. 83 (2019) 572-575.

[5] V.V. Shutenko et al. Observation of heliospheric disturbances in the muon component of cosmic rays, Bull. Rus. Acad. Sci. Phys. 73 (2009) 347-349.

[6] OMNI database: omniweb.gsfc.nasa.gov. 